\title{
Plastic stents are more cost-effective than lumen-apposing metal stents in management of pancreatic pseudocysts
}

\section{(ㄷ)(1) $\odot$}

Authors

Yen-I Chen ${ }^{1,2}$, Mouen A. Khashab ${ }^{1}$, Viviane Adam², Ge Bai ${ }^{3}$, Vikesh K. Singh¹, Majidah Bukhari ${ }^{1}$, Olaya Brewer Gutierrez ${ }^{1}$, B. Joseph Elmunzer', Robert A. Moran ${ }^{1}$, Lea Fayad ${ }^{1}$, Mohamad El Zein ${ }^{1}$, Vivek Kumbhari ${ }^{1}$, Alessandro Repici ${ }^{5}$, Alan N. Barkun²

\section{Institutions}

1 Division of Gastroenterology and Hepatology, Johns Hopkins Medical Institutions, Baltimore, Maryland, United States

2 Division of Gastroenterology and Hepatology, The McGill University Health Center, Montreal, Quebec, Canada

3 Johns Hopkins Carey Business School, Baltimore, Maryland, United States.

4 Division of Gastroenterology and Hepatology, Medical University of South Carolina, Charleston, South Carolina, United States

5 Humanitas University, Milan, Italy

submitted 28.2.2018

accepted after revision 13.4 .2018

\section{Bibliography}

DOI https://doi.org/10.1055/a-0611-5082 |

Endoscopy International Open 2018; 06: E780-E788

(c) Georg Thieme Verlag KG Stuttgart · New York

ISSN 2364-3722

Corresponding author

Dr. Alan Barkun, Room D7-346, Division of

Gastroenterology, McGill University Health Centre, Montreal General Hospital site, 1650 Cedar Avenue,

Montréal, Québec, H3G 1A4, Canada

Fax: +1-514-934-8375

alan.barkun@muhc.mcgill.ca

\section{ABSTRACT}

Background and study aims Endoscopic ultrasoundguided drainage is an effective and accepted primary modality for management of pancreatic pseudocyst (PP). A lumen-apposing metal stent (LAMS) has recently been developed specifically for drainage of pancreatic fluid collections which may be superior to using traditional plastic stents (PS) but is more expensive. Because use of a stent involves a risk of unplanned endoscopy, percutaneous drainage (PCD) and surgery, their costs should also be included in the comparison and a cost-effectiveness analysis of LAMS and PS should therefore be performed

Patients and methods A decision tree was developed assessing both endoscopic drainage strategies for patients with PP: LAMS and PS over a 6-month time horizon. For each strategy, inpatients received a stent and were followed for subsequent need for direct further interventions or adverse events leading to unplanned endoscopy, PCD, surgery, or successful endoscopic drainage using probabilities obtained from the literature. The unit of effectiveness was successful endoscopic drainage without need for PCD or surgery. Sensitivity analyses were performed.

Results Success rates were $93.9 \%$ for LAMS and $96.96 \%$ for PS. Respective costs per successful drainage were US $\$ 18,129$ (LAMS) and US $\$ 10,403$ (PS). The LAMS strategy was thus characterized as dominated by the PS approach because it was costlier and less effective than PS. Both deterministic and probabilistic sensitivity analyses confirmed the robustness of these findings.

Conclusion Use of LAMS is not less effective and more costly than PS in management of patients with PP. As such, PS should be preferred over LAMS as initial management of these patients.

\section{Introduction}

Pancreatic pseudocysts (PP) are common local complications of pancreatitis that can be associated with significant morbidity [1-2]. According to the revised Atlanta classification [3], PP is defined as a mature fluid collection with a well-defined wall without solid components usually developing 4 weeks postpancreatitis. Although generally asymptomatic and self-resolving, drainage of PP is required if there are persistent symptoms such as early satiety, abdominal pain, or gastric outlet obstruction despite adequate conservative management or when there is evidence of fluid collection infection [4]. 
Endoscopic drainage with insertion of double pigtail plastic stents has been shown to be effective and safe and has gained popularity with the evolving field of therapeutic endoscopic ultrasound (EUS). Recently, lumen-apposing metal stents (LAMS) have been developed specifically for drainage of pancreatic fluid collections [5]. Advantages of LAMS include their large diameter $(10-15 \mathrm{~mm})$, bi-flared flanges preventing stent migration, and ease of insertion as one-step devices including cautery without need for needle puncture, wire guidance, tract dilation, or fluoroscopic guidance. Prospective and retrospective series have shown excellent efficacy and safety of LAMS in management of PP [6-15]; however, LAMS are also known as considerably more expensive than traditional plastic stents (PS). Insertion of a stent could also generate possible adverse events and therefore the need for unplanned endoscopy, percutaneous drainage (PCD) or even surgery that should be taken into account in the comparison. There are currently no published cost-effectiveness data on use of LAMS in PP to inform optimal resource allocation. The objective of this work was to determine whether the advantages in terms of cost and effectiveness in favor of LAMS (reduced rate of complication) are outweighed by those in favor of PS (considering its success rate and lower stent price).

It is with this intricate backdrop in mind that this study aimed to assess the cost-effectiveness of EUS-guided drainage using LAMS (AXIOS, Boston Scientific, Marlborough, Massachusetts, United States) compared to PS in management of pancreatic pseudocysts.

\section{Patients and methods}

\section{Model design}

A decision model was constructed to assess clinical aspects of management of PP comparing LAMS to PS in inpatients over a 6 -month period following stent insertion. This type of analysis was chosen to simultaneously evaluate cost and effectiveness of the traditional PS versus the novel LAMS. - Fig. 1 shows that at the initial node, all inpatients receive a stent: either LAMS or PS. If this procedure fails, patients undergo PCD leading directly to a terminal node as a general assumption of the model is that any second modality used following initial drainage failure is successful. Otherwise, patients are followed for subsequent complication resulting in a need fir unplanned endoscopy, PCD or surgery. In the case of an unplanned endoscopy, a salvage plastic stent is assumed to be inserted in both LAMS and PS groups. Death as possible choice for unit of effectiveness was not considered in the model due to the lack of evidence to suggest differences in survival between PS and LAMS in the literature and the short but clinically appropriate chosen time horizon of 6 months $[6,8-16]$. The unit of effectiveness was defined at the end of each path as the successful endoscopic drainage rate without need for PCD or surgery. The total cumulative representative costs across the time horizon were also computed for each path in the tree. This allows presentation of the outcome at each terminal node as the average individual total cost per patient drained successfully at the term of 6 months. Physician fees and hospital costs, including procedure costs and pharmaceutical costs, were considered in the context of a third-party-payer perspective. The software program TreeAge Pro Suite 2017 was used for this model and analysis [17].

\section{Probabilities}

\section{Search strategy}

Probabilities were derived from the literature. A computerized medical literature search was performed using PubMed, the Cochrane library, and Embase from January 1992 until September 2016. Only fully published manuscripts in English were included. A highly sensitive search strategy was used to identify randomized, retrospective and prospective studies using the following keywords and MESH terms: pancreatitis, pancreatic pseudocyst, necrotizing pancreatitis, peripancreatic fluid collection, pancreatic fluid collection, acute necrotic collection, acute necrotizing collection, walled-off necrosis (WON), pancreatic abscess, pancreatic necrosis, EUS guided drainage, endoscopic ultrasound-guided drainage, endoscopic debridement, necrosectomy, endoscopic transmural drainage, sinus tract endoscopy, video-assisted retroperitoneal debridement, pseudocyst, percutaneous drainage. The search then focused on transmural drainage by using the keywords: 1) Transmural AND drainage (search all references), 2) EUS guided transmural drainage (search transmural drainage references) (Endoscopic; EUS; endoscopy) AND (ultrasound; ultrasonography), 3) Direct endoscopic necrosectomy (search all references), Endoscopic AND necrosectomy. In addition, recursive searches and crossreferencing were performed, as were hand searches of articles identified after the initial search was completed.

\section{Study selection and patient population}

We included retrospective and prospective studies in English involving endoscopic drainage of pancreatic PP using LAMS (AXIOS LAMS Boston Scientific, Marlborough, Massachusetts, United States) or PS. Only fully published studies were included. Excluded articles were those that reported drainage of WON, review articles, articles with less than 10 cases, studies for which pseudocyst and pancreatic WON were not differentiated as per the Atlanta classification [3], those addressing non EUSguided endoscopic drainage, drainage performed with traditional esophageal or biliary metal stents, and drainage with SPAXUS or NAGI LAMS (Taewoong Medical, South Korea) [18]. All probabilities were calculated from a weighted average (per sample size) of the available literature.

\section{Study endpoints}

Primary outcomes were technical and clinical success. Technical success was defined as successful stent insertion. Although successful drainage varied from study to study, we adopted a broad definition of symptomatic and radiographical resolution following stent insertion. Secondary outcomes included adverse events requiring unplanned endoscopic procedures, as well as the need for percutaneous drainage and surgery. 


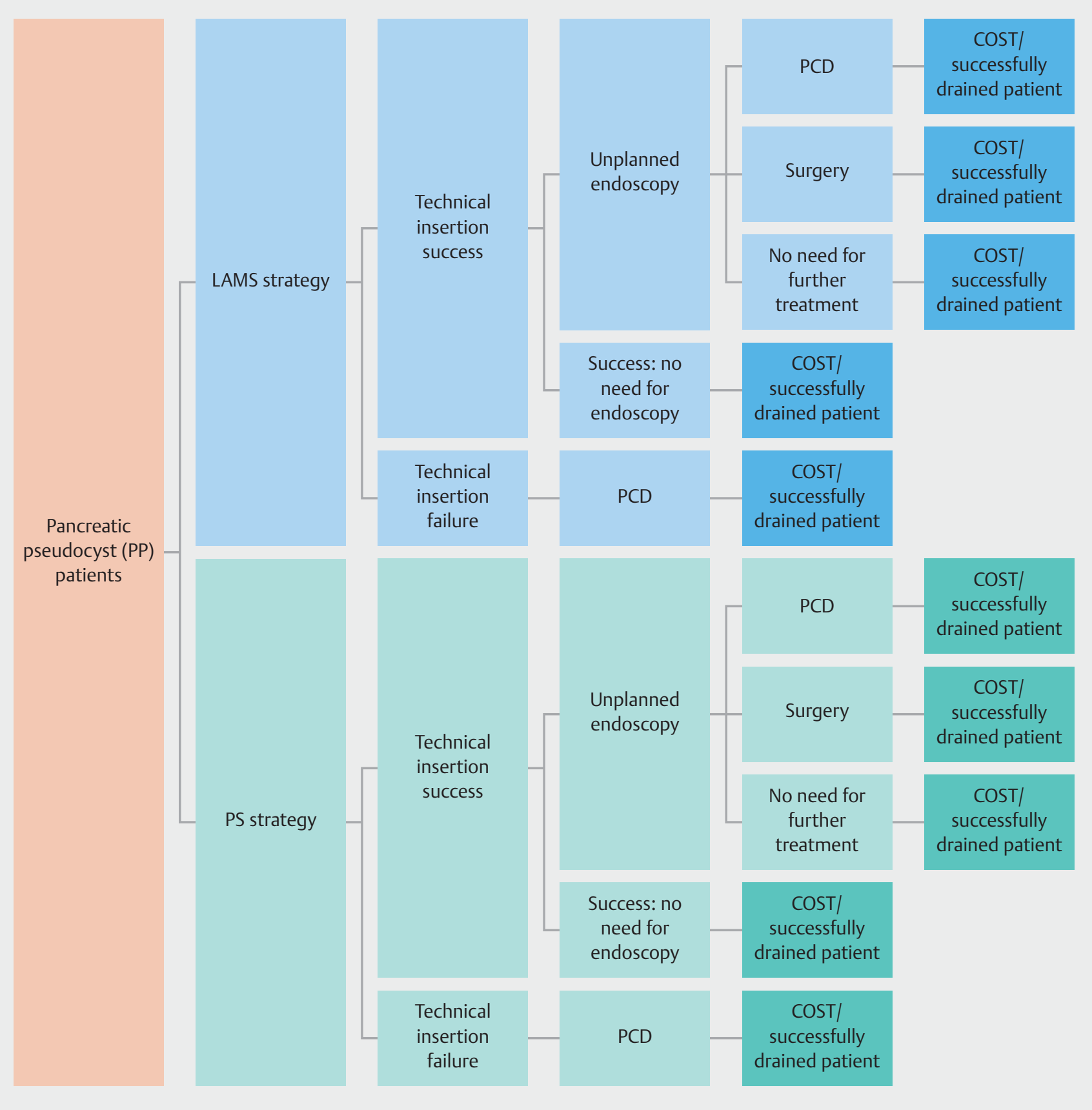

- Fig. 1 Model structure. Time horizon was 6 months. LAMS, lumen-apposing metal stent; PP, pancreatic pseudocyst; PS, plastic stent; PCD, percutaneous drain.

\section{Costs and lengths of stay}

Costs for the analysis were calculated using billing claims at Johns Hopkins Hospital [19]. Inpatients who underwent endoscopic drainage or PCD of peripancreatic fluid collections between January 2012 and March 2016 were identified using procedure codes 0F9G4ZZ (drainage of pancreas, percutaneous endoscopic approach), 0F9G40Z (Drainage of pancreas with drain device, percutaneous endoscopic approach), and 5201 (drainage of pancreatic cyst by catheter). Overall and otherwise unselected, 12 patients who underwent drainage with a plastic stent, 14 patients with a LAMS, and four patients who underwent percutaneous drainage were identified. Mean charges for operating room (for index procedure, unplanned endoscopy, direct endoscopic necrosectomy), supply including stent prices, pharmacy, radiology, laboratory, therapy, other, and total were ascertained. Length of stay and associated charges were also obtained from the Johns Hopkins Hospital billing database; however, given the lack of patients who underwent surgery in our cohort, data on post-surgical LOS were obtained from the 
- Table 1 Probabilities.

\begin{tabular}{|l|l|l|l|l|l|l|l|l|}
\hline \multirow{2}{*}{ Description of probability } & \multicolumn{3}{|l|}{ LAMS strategy } & \multicolumn{2}{|c|}{ PS strategy } \\
\hline & Baseline & Low & High & Source & Baseline & Low & High & Source \\
\hline Technical stent insertion success & 0.9412 & 0.75 & 1 & {$[6,10,14,15]$} & 0.9761 & 0.78 & 1 & {$[6,8,9,11-13,16]$} \\
\hline Unplanned endoscopy & 0.1176 & 0.09 & 0.15 & {$[6,10,14,15]$} & 0.1226 & 0.09 & 0.15 & {$[6,8,9,11-13,16]$} \\
\hline PCD & 0 & 0 & 0.05 & {$[6,10,14,15]$} & 0.0068 & 0 & 0.05 & {$[8,9,12,13,16]$} \\
\hline Surgery & 0.0196 & 0.01 & 0.05 & {$[6,10,14,15]$} & 0.0473 & 0.03 & 0.06 & {$[8,9,12,13,16]$} \\
\hline
\end{tabular}

literature [20]. Charges were converted into costs using the Johns Hopkins Hospital's overall cost-to-charge ratio in the fiscal period when the procedure was performed. Indeed, we obtained cost-to-charge ratios from the Centers for Medicare and Medicaid Services [21] and Hospital Cost reports (HCR) [22]. Costs covered a period spanning 2012 to 2016; for procedures performed after July 1,2015 , the average charge-to-cost ratio across 2011 to 2014 was used. None of the 14 patients identified underwent surgical intervention. Therefore, institutional cost data were not available for surgical management of peripancreatic fluid collections. As such, estimates of surgical costs were ascertained using published literature, which also provided the physician fees for anesthesia [20]. All other professional/ physician fees for endoscopic drainage, percutaneous drainage, and surgical cystgastrostomy were estimated using the average state fees of Maryland provided by the American Medical Association [23]. Hospital per diem costs were computed as the ratio of the cost per hospitalization to the associated LOS. Discounting was not applied due to the short time horizon. All costs were expressed in 2016 US dollars utilizing the consumer price index for the medical care services published by the United States Department of Labor [24].

\section{Cost-effectiveness analysis}

The effectiveness is expressed as the probability of successful endoscopic drainage without need for PCD or surgery over the full duration of the model. The costs are the sum of the cost items related to each event in the model. The outcome is the cost per patient drained successfully across the time horizon. Results are reported as cost, effectiveness, cost-effectiveness, and incremental cost effectiveness ratios.

\section{Sensitivity analyses}

Both deterministic and probabilistic sensitivity analyses were undertaken. One-way sensitivity analyses on all variables of the models were done to assess robustness of the results. Sensitivity analyses allowed observation of how the results could change if the input variables included in the model were different than those chosen. We varied the value of each of the parameters of the model inside their respective pre-fixed ranges while other input variables were kept unchanged. Threshold analysis was also performed across plausible ranges on all variables to determine which variables could alter the conclusion. In addition, probabilistic sensitivity analysis was performed using a second-order Monte Carlo simulation running 10,000 variations of the model varying simultaneously all resampled parameters according to their statistical distributions [25].

Cost-acceptability curve and incremental cost-effectiveness scatterplot (cloud diagram) were generated, adopting a willingness-to-pay threshold of US $\$ 50,000$ per successful outcome as we have done previously for such analyses [26, 27]. A willingness to pay (WTP) represents the pre-fixed maximum dollar value that is deemed as acceptable spending for a given treatment. It illustrates the probability that one type of stent is cost-effective compared the other one, given a WTP value.

The reporting of our results follows the Consolidated Health Economic Evaluation Reporting Standards Statement [28].

\section{Results}

\section{Probability and cost assumptions}

A total of 5400 articles were identified and screened for potential inclusion. Following the removal of duplicates and the aforementioned exclusion criteria, 10 fully published manuscripts were included in the systematic review for the determination of probability assumptions ( Appendix 1 ) [6,8-16]. These included nine retrospective studies and one prospective series. No randomized controlled trials were identified. There were a total of 51 patients in the LAMS cohort and 377 patients in PS group. Probabilities obtained from these studies are summarized in > Table 1.

All probabilities and their respective sources used in the model are listed in $>$ Table 1, while LOS and costs are shown in - Table2. The lower and upper limits of the intervals were set to $\pm 20 \%$ of their respective baseline values for the probabilities and to $\pm 50 \%$ for the costs. Probabilities followed beta and costs gamma distributions [29].

\section{Base-case analysis}

- Table 3 shows the overall cost-effectiveness analysis report according to the type of stent chosen to manage PP over 6 months. Inserting a LAMS would generate an average cost of US $\$ 17,024$ per patient in comparison with US $\$ 10,087$ for a PS. The success rate was $93.9 \%$ for LAMS and $96.96 \%$ for PS. Respective costs per successful drainage were US $\$ 18,129$ (LAMS) and US $\$ 10,403$ (PS). Being both more costly and less effective, 
- Table 2 Length of stay and costs.

\begin{tabular}{|c|c|c|c|c|c|}
\hline Category & Description & Baseline $^{1}$ & Low ${ }^{1}$ & High $^{1}$ & Source \\
\hline LOS & TIS/Unplanned endoscopy/PCD/Tn & 6 & 3 & 9 & Billing claims [19] \\
\hline LOS & TIS/Unplanned endoscopy/surgery/Tn & 10 & 5 & 15 & Billing claims [19] \\
\hline LOS & TIS/Unplanned endoscopy/Successfully drained patient/Tn & 4 & 2 & 6 & Billing claims [19] \\
\hline LOS & TIS/Successfully drained patient/Tn & 2 & 1 & 3 & Billing claims [19] \\
\hline LOS & Technical failure at the insertion/PCD/Tn & 4 & 2 & 6 & Billing claims [19] \\
\hline Per diem & Per diem hospital cost & 2437 & 1218 & 3656 & Billing claims [19] \\
\hline Price & PS & 104 & 52 & 156 & Billing claims [19] \\
\hline Price & LAMS & 4930 & 2465 & 7395 & Billing claims [19] \\
\hline Cost procedure & PS insertion & 3272 & 1636 & 4908 & Billing claims [19] \\
\hline Cost procedure & LAMS insertion & 5237 & 2618 & 7856 & Billing claims [19] \\
\hline Cost procedure & TIS/Unplanned endoscopy & 3786 & 1893 & 5679 & Billing claims [19] \\
\hline Cost procedure & Surgery & 2136 & 1068 & 3204 & Varadarajulu $[20,24]$ \\
\hline Cost procedure & PCD & 3629 & 1814 & 5444 & Billing claims [19] \\
\hline Physician fees & Stent insertion & 443 & 221 & 665 & CPT43240, [23] \\
\hline Physician fees & TIS/Unplanned endoscopy & 203 & 101 & 305 & CPT43247, [23] \\
\hline Physician fees & Surgery & 3154 & 1577 & 4731 & CPT48105, [23] \\
\hline Physician fees & PCD & 231 & 115 & 347 & CPT49406, [23] \\
\hline Physician fees & Anesthesia & 712 & 356 & 1068 & Varadarajulu $[20,24]$ \\
\hline
\end{tabular}

LAMS, lumen-apposing metal stent; LOS, length-of-stay; PCD, percutaneous drain; PS, plastic stent; TIS, technical insertion success; Tn, terminal node

${ }^{1}$ Costs are expressed in 2016 US $\$$ and LOS are expressed in days.

- Table 3 Cost-effectiveness report.

\begin{tabular}{|l|l|l|l|l|l|l|}
\hline Strategy & Cost $^{\mathbf{1}}$ & IC & Effectiveness & IE & CER \\
\hline PS & 10087 & & 0.9696 & & 10403 \\
\hline LAMS & 17024 & 6937 & 0.939 & -0.0306 & 18129 & Dominated \\
\hline
\end{tabular}

${ }^{1}$ Costs are expressed in 2016 US \$. Effectiveness is expressed as rate of successfully drained patient. CER, cost-effectiveness ratio; IC, incremental cost; CER, incremental cost-effectiveness ratio; IE, incremental effectiveness; LAMS, lumen-apposing metal stent; PS, plastic stent

the LAMS strategy was thus characterized as dominated (in the economic sense) by the PS approach.

\section{Sensitivity analysis}

According to one-way sensitivity analysis, only the success rate of stent insertion could change the final preferred strategy. If the rate of success increased above $97.2 \%$ for LAMS, or decreased below $94.5 \%$ for PS, LAMS no longer dominated. The threshold analysis showed that other possible threshold values for changing the conclusions lie far outside the pre-set ranges of clinically relevant assumptions. This includes stent prices, which would not alter our conclusion even when the price of LAMS was varied from US $\$ 0$ to US $\$ 20,000$ while keeping the price of PS constant at US $\$ 104$. In addition, probabilities associated with the PS strategy of insertion success (more than $97 \%$ ), PCD (less than $1 \%$ ) or surgery (less than $5 \%$ ) should respectively decrease below $68 \%$, rise above $26 \%$ and above $30 \%$ for the LAMS strategy to no longer be dominated.

Probabilistic sensitivity analysis confirmed the robustness of the base case results. LAMS was dominated by PS in $74 \%$ of simulation scenarios, while the LAMS approach preferred over PS in only $7.3 \%$. Overall, PS was chosen (more cost-effective or actually dominating LAMS) in $92.7 \%$ of all simulations. P Fig. 2 presents the incremental cost-effectiveness scatterplot with a WTP line drawn at US $\$ 50,000$ : The point of origin $(0 ; 0)$ represents the PS strategy as reference. Each point of the cloud represents a difference between the PS and LAMS strategies from a point of view of both effectiveness and cost for every clinical scenario generated by the Monte Carlo Analysis. The ellipse that includes $95 \%$ of all simulation points is to the left of the graph and slightly in the top quadrant, confirming that the LAMS strategy is most often less effective and more costly than 


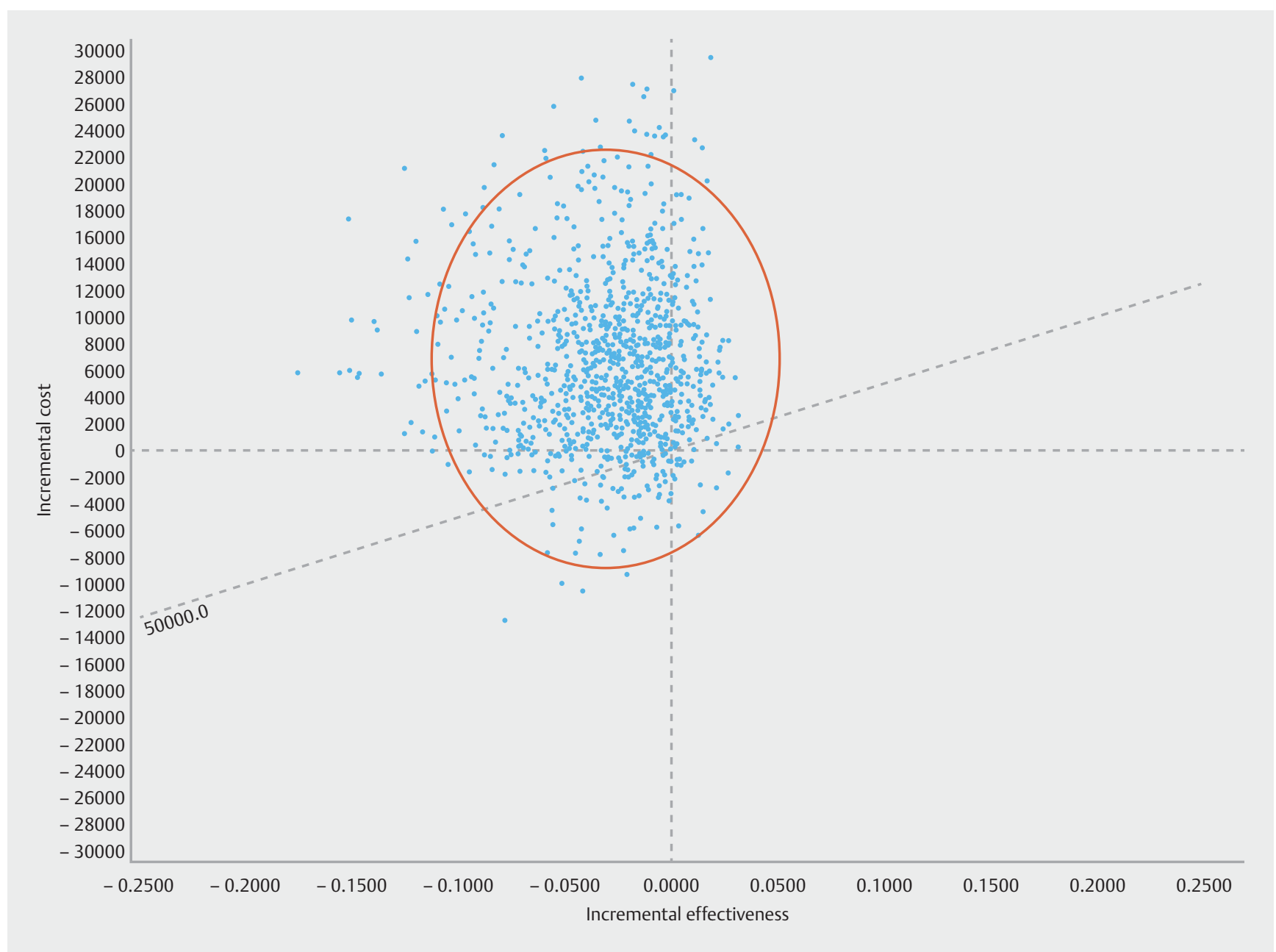

- Fig. 2 Incremental cost-effectiveness scatterplot LAMS versus PS. Costs are expressed in US \$. LAMS, lumen-apposing metal stent; PS, plastic stent; WTP, willingness to pay (Line displayed at US $\$ 50,000$.)

the PS strategy using such a probabilistic assessment. The costacceptability curve ( $\bullet$ Fig. $\mathbf{3}$ ) highlights how the value set for the WTP would not change the conclusion.

\section{Discussion}

Endoscopic-guided transmural drainage of pancreatic pseudocysts has gained popularity with the evolving field of therapeutic EUS. The advent of LAMS has created great excitement in this field with several studies demonstrating their excellent efficacy and safety in management of pancreatic fluid collections $[6,14,30,31]$. The major disadvantage associated with LAMS are their high cost. To our knowledge, this is the first cost-effectiveness analysis comparing LAMS with PS in management of $\mathrm{PP}$, which is timely given that many endoscopy centers worldwide are contemplating adopting these devices. Our results would be generalizable for countries with similar proportional cost structure with cost estimates falling within our ranges.

In this study, use of LAMS appeared to be dominated by PS in management of pancreatic pseudocysts meaning that use of LAMS is not more beneficial while being costlier than PS. These results are robust and are consistent throughout extensive de- terministic and probabilistic sensitivity analyses, meaning that our conclusion does not alter despite considering large variations in probability and costs assumptions.

Favorable stent characteristics of LAMS including their larger luminal diameter and bi-flared flanges preventing stent migration do not appear to provide significant incremental benefits over PS in PP-probably given the absence of solid debris and therefore low likelihood of plastic stent obstruction. The same could not be said of pancreatic WON, defined as a mature pancreatic collection with solid debris, where risk for stent obstruction is high and where a larger stent diameter along with antimigration properties to allow for direct endoscopic necrosectomy through the stent provide significant additional improvements in outcome. Indeed, retrospective comparative studies have suggested that LAMS may be more effective than PS in WON [32]. As a result, it is not surprising that a recent cost analysis, by our group, suggested LAMS are cost-effective when compared to PS in the endoscopic drainage of WON [33]. Overall, our data reaffirm the need for proper diagnosis and classification of pancreatic fluid collections to select the most appropriate management approach. For optimal resource allocation, it appears that LAMS are best reserved for WON while the use of 


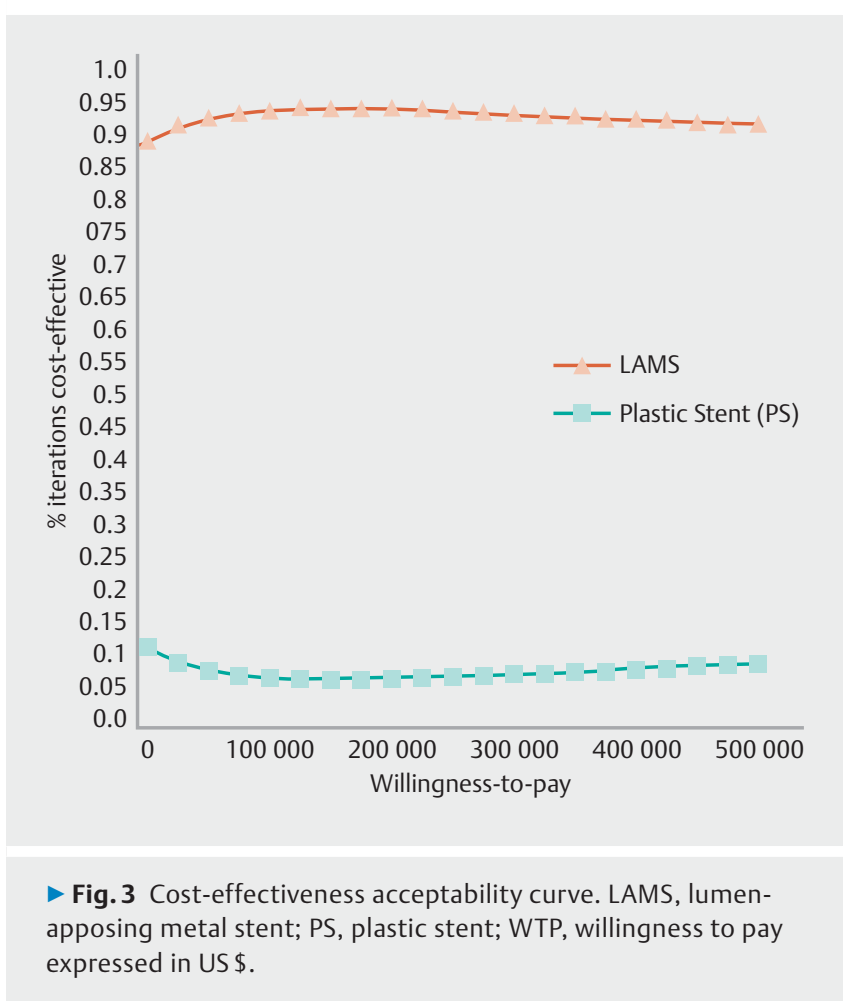

PS is likely the better choice in pancreatic pseudocysts. Many endoscopists nevertheless will likely opt for use of LAMS over PS in PP given their ease of insertion, especially in community hospitals where fluoroscopy access may be limited. However, in tertiary centers where endoscopic expertise is available and where fluoroscopic assistance is readily accessible, PS is likely the better and more cost-effective modality for PP.

There are several important aspects of our study that merit further discussion. First, we did not use Quality Adjusted Life Years (QALYs) as the measure of effectiveness as for acute medical conditions such as pancreatic pseudocysts, better adapted clinical endpoints including successful endoscopic drainage may be a more appropriate alternative to optimally inform resource allocation [34,35]. In addition, death was not included in the analysis given its low prevalence in this patient population, lack of data to suggest any differences between the two groups in the literature, [6,8-16], and the adopted short study time-horizon, as similarly justified in past cost-effectiveness models addressing endoscopic hemostasis [26]. In clinical practice the majority of pseudocyst recurrence occurs within a few months following clinical success. Considering that the most relevant direct and measurable consequences occur in the first months following insertion and considering the lack of longterm data in the literature, a time horizon of 6 months was adopted. Limitations of the study include the fact that the decision tree may be oversimplified, although it was constructed in a manner to be as comprehensive as possible and clinically relevant, with the literature informing the different assumptions chosen for the health states. Also, only inpatient costs were included to have a more homogenous patient population with more conservative assumptions; however, this may limit the generalizability of results to outpatients. In terms of probability assumptions, there were no controlled trials available in the literature and data were obtained from retrospective and prospective series, which likely led to an overestimation of technical and clinical success with both LAMS and PS. Inherent biases in these studies may also explain the surprising finding that PS was slightly more effective than LAMS. In our sensitivity analysis, however, LAMS would only no longer be dominated if drainage success, need for PCD, and need for surgery with PS should respectively decrease below $68 \%$, rise above $26 \%$, and above $30 \%$. As such, biases in the literature are unlikely to affect our results, which appear to be robust. As for costs, the generalizability of the chosen estimates may be limited as only a single institution was used for source data while only the Maryland AMA information was used for professional fees. An exploratory analysis we carried out (data available upon request) suggest that using the national US average would have given a maximal variation of $10 \%$ of the values we used for Maryland. These variations were thus included in the ranges we used for the sensitivity analysis. Similarly, cost data for surgical intervention were obtained from the literature as no institutional data were available [20]. Lastly the analysis did not include use of traditional fully-covered metal biliary or esophageal stents as well as other LAMS such as the NAGI or SPAXUS stents. Considering the wide ranges of assumptions used for sensitivity analysis of all cost data, as shown in $>$ Table 2, these limitations were thus once again likely accounted for and do not change our conclusions.

\section{Conclusion}

In conclusion, use of PS is more effective than LAMS while being also less costly in management of pancreatic pseudocysts. As such, PS should be preferred over LAMS as initial management of patients with PP.

\section{Competing interests}

Dr Chen, Dr Kumhari and Dr Repici and Dr Khashab served as consultants for Boston Scientific. Dr Singh is a consultant for Abbvie, Akcea, and Novo Nordisk and an advisory board participant for Nordmark. Dr Barkun served as a consultant for Cook Inc, Pendopharm Inc., and Olympus (also research support and advisory committee) and received funds from Cook Inc., Pendopharm Inc. and Olympus. He had full access to all data in the study and takes responsibility for the integrity of the data and the accuracy of the data analysis.

\section{References}

[1] Poornachandra KS, Bhasin DK, Nagi B et al. Clinical, biochemical, and radiologic parameters at admission predicting formation of a pseudocyst in acute pancreatitis. J Clin Gastroenterol 2011; 45: 159-163

[2] Tenner S, Baillie J, DeWitt J et al. American College of Gastroenterology guideline: management of acute pancreatitis. Am J Gastroenterol 2013; 108: $1400-1415 ; 1416$ 
[3] Banks PA, Bollen TL, Dervenis C et al. Classification of acute pancreatitis-2012: revision of the Atlanta classification and definitions by international consensus. Gut 2013; 62: 102-111

[4] Muthusamy VR, Chandrasekhara V. Committee ASoP. et al. The role of endoscopy in the diagnosis and treatment of inflammatory pancreatic fluid collections. Gastrointest Endosc 2016; 83: 481-488

[5] Binmoeller KF, Shah J. A novel lumen-apposing stent for transluminal drainage of nonadherent extraintestinal fluid collections. Endoscopy 2011; 43: $337-342$

[6] Bang JY, Hasan MK, Navaneethan U et al. Lumen-apposing metal stents for drainage of pancreatic fluid collections: When and for whom? Dig Endosc 2017; 29: 83 - 90

[7] Chaves DM, Monkemuller K, Carneiro F et al. Endoscopic treatment of large pancreatic fluid collections (PFC) using self-expanding metallic stents (SEMS) - a two-center experience. Endosc Int Open 2014; 2: E224-E229

[8] Giovannini M, Pesenti C, Rolland AL et al. Endoscopic ultrasoundguided drainage of pancreatic pseudocysts or pancreatic abscesses using a therapeutic echo endoscope. Endoscopy 2001; 33: 473-477

[9] Heinzow HS, Meister T, Pfromm B et al. Single-step versus multi-step transmural drainage of pancreatic pseudocysts: the use of cystostome is effective and timesaving. Scand J Gastroenterol 2011; 46: $1004-1013$

[10] Itoi T, Binmoeller KF, Shah J et al. Clinical evaluation of a novel lumenapposing metal stent for endosonography-guided pancreatic pseudocyst and gallbladder drainage (with videos). Gastrointest Endosc 2012; 75: 870-876

[11] Lin H, Zhan XB, Sun SY et al. Stent selection for endoscopic ultrasound-guided drainage of pancreatic fluid collections: a multicenter study in china. Gastroenterol Res Pract 2014; 2014: 193562

[12] Ng PY, Rasmussen DN, Vilmann P et al. Endoscopic ultrasound-guided drainage of pancreatic pseudocysts: medium-term assessment of outcomes and complications. Endosc Ultrasound 2013; 2: 199-203

[13] Rische S, Riecken B, Degenkolb J et al. Transmural endoscopic necrosectomy of infected pancreatic necroses and drainage of infected pseudocysts: a tailored approach. Scand J Gastroenterol 2013; 48: $231-240$

[14] Siddiqui AA, Adler DG, Nieto J et al. EUS-guided drainage of peripancreatic fluid collections and necrosis by using a novel lumen-apposing stent: a large retrospective, multicenter U.S. experience (with videos). Gastrointest Endosc 2016; 83: 699-707

[15] Walter D, Will U, Sanchez-Yague A et al. A novel lumen-apposing metal stent for endoscopic ultrasound-guided drainage of pancreatic fluid collections: a prospective cohort study. Endoscopy 2015; 47: $63-67$

[16] Varadarajulu S, Bang JY, Phadnis MA et al. Endoscopic transmural drainage of peripancreatic fluid collections: outcomes and predictors of treatment success in 211 consecutive patients. J Gastrointest Surg 2011; $15: 2080-2088$

[17] TreeAge Software Inc. Healthcare Module. Williamstown, MA, USA: 2017: Available at: https://www.treeage.com/ [Accessed March, 2018]

[18] Taewoong Medical. Available at: http://www.stent.net/ [Accessed March, 2018]
[19] Johns Hopkins Hospital. Available at: http://intranet.insidehopkinsmedicine.org/finance/departments/revenue-reimbursement/informatics.html [Accessed April, 2018]

[20] Varadarajulu S, Bang JY, Sutton BS et al. Equal efficacy of endoscopic and surgical cystogastrostomy for pancreatic pseudocyst drainage in a randomized trial. Gastroenterology 2013; 145: 583 - 590 e581

[21] Centers for Medicare and Medicaid Services. Available at: https:// www.cms.gov/ [Accessed Jan, 2018]

[22] Hospital Form 2552-10. Baltimore (MD): Centers for Medicare and Medicaid Services; 2015: Available at: https://www.cms.gov/Research-Statistics-Data-and-Systems/Downloadable-Public-Use-Files/ Cost-Reports/Hospital-2010-form.html [Accessed March, 2018]

[23] American Medical Association. Available at: http://www.ama-assn. org/ [Accessed March, 2018]

[24] U.S. Bureau of Labor Statistics. Available at: http://www.bls.gov/ [Accessed Janv, 2018]

[25] Briggs A. Decision modelling for health economic evaluation. Oxford University Press ed. Oxford University Press; 2006

[26] Barkun AN, Adam V, Lu Y et al. Using Hemospray improves the costeffectiveness ratio in the management of upper gastrointestinal nonvariceal bleeding. J Clin Gastroenterol 2018; 52: 36 - 44

[27] Barkun AN, Adam V, Martel M et al. Partially covered self-expandable metal stents versus polyethylene stents for malignant biliary obstruction: a cost-effectiveness analysis. Can J Gastroenterol Hepatol 2015; 29: $377-383$

[28] Husereau D, Drummond M, Petrou S et al. Consolidated Health Economic Evaluation Reporting Standards (CHEERS) statement. Value Health 2013; 16: e1 - 5

[29] Neumann PJ, Sanders GD, Russel LB et al. Cost-effectiveness in health and medicine. 2nd ed. Oxford University Press; 2017

[30] Chandran S, Efthymiou M, Kaffes A et al. Management of pancreatic collections with a novel endoscopically placed fully covered self-expandable metal stent: a national experience (with videos). Gastrointest Endosc 2015; 81: 127 - 135

[31] Sharaiha RZ, Tyberg A, Khashab MA et al. Endoscopic therapy with lumen-apposing metal stents is safe and effective for patients with pancreatic walled-off necrosis. Clin Gastroenterol Hepatol 2016; 14: $1797-1803$

[32] Siddiqui AA, Kowalski TE, Loren DE et al. Fully covered self-expanding metal stents versus lumen-apposing fully covered self-expanding metal stent versus plastic stents for endoscopic drainage of pancreatic walled-off necrosis: clinical outcomes and success. Gastrointest Endosc 2017; 85: $758-765$

[33] Chen Y, Barkun AN, Adam V et al. Sa 1402 Lumen apposing metal stents are cost-effective when compared with plastic stents in the management of pancreatic walled-off necrosis. Gastrointest Endosc [Abstract] 2017; 85: AB227

[34] Bala MV, Zarkin GA. Are QALYs an appropriate measure for valuing morbidity in acute diseases? Health Econ 2000; 9: 177-180

[35] Franic DM, Pathak DS, Gafni A. Quality-adjusted life years was a poor predictor of women's willingness to pay in acute and chronic conditions: results of a survey. J Clin Epidemiol 2005; 58: 291 - 303 
5400 references for screening

\section{1 duplicates}

3129 studies for abstract and title screening

2405 not related to PP or review article

202 abstracts only

181 not endoscopic drainage of PP

98 case reports

80 published prior to 1992

74 other

89 studies for full-text screening

79 WON, undefined collection, non-EUS guided,

Spaxus/NAGI stent, or biliary or esophageal metal stent

10 studies included

\section{Appendix 1}

